\title{
Isolation and characterization of DNA barcodes from distinctive and rare terrestrial animals in China using universal $\mathrm{COI}$ and $16 \mathrm{~S}$ primers
}

\author{
Mali GUO a,b,\#, Zhenzhen PENG ${ }^{a, b, \#}$, Xiaoting ZHANG ${ }^{a, b, \#}$, Chaohai YUAN ${ }^{a, b}$, Hanxi LU ${ }^{a, b}$, Keyun \\ ZHANG $^{\mathrm{d}}$, Yafei CAl ${ }^{\mathrm{a}, \mathrm{b}, *}$, Wei ZHANG $^{\mathrm{a}, \mathrm{b}, *}$ \\ ${ }^{1}$ College of Animal Science and Technology, Nanjing Agricultural University, 210095 Nanjing, China \\ ${ }^{2}$ National Experimental Teaching Demonstration Center of Animal Science, Nanjing Agricultural University, 210095 Nanjing, China \\ ${ }^{3}$ College of Life Science, Nanjing Agricultural University 210095 Nanjing, China \\ \# These authors contribute equally. \\ *Correspondence: weizhang@njau.edu.cn, ycai@njau.edu.cn \\ https://doi.org/10.37175/stemedicine.v2i7.95
}

\begin{abstract}
Background: Accurate taxonomic identification is the cornerstone for monitoring, conservation and management of ecological resources. China has the highest biodiversities and the richest species assemblages in the world, but is lacking in sufficient assessment to the abundant genetic variability. DNA barcoding is a proven tool employing sequence information for rapid and unambiguous species delineation. However, the ability of barcodes to distinguish species that are archaic and distinctive evolutionary lines remains largely untested.

Methods: In order to investigate the resources of terrestrial animals in China, regions from mitochondrial $\mathrm{CO}$ and $16 \mathrm{~S}$ are barcoded for 395 specimens belonging to 54 selected species, many of which are indigenous representatives in danger. High success rate of PCR amplification is achieved by using universal $\mathrm{COI}$ and $16 \mathrm{~S}$ primers with many numts pseudogenes co-amplified from mammalian samples.

Results: Application of barcodes to flag species is generally straightforward since no $\mathrm{CO}$ or $16 \mathrm{~S}$ haplotypes are shared between closely related species. Barcoding gap, species resolution and phylogenetic relationships relying on our barcode libraries are further compared using distance and tree based approaches.

Conclusion: Results show that the discriminatory power of the two barcode markers could differentiate on a case-by-case basis, and also suggest a careful consideration of the nuclear numts for barcoding studies as they might provide a new understanding for evolution.
\end{abstract}

Keywords: DNA barcode $\cdot \mathrm{COI} \cdot 16 \mathrm{~S} \cdot$ Indigenous animals $\cdot$ China

\section{Introduction}

China is among the highest biodiversities and has the richest species assemblages in the world. It is estimated that over $10 \%$ of the world's ecosystem types exist in this country, including $\sim 2485$ species of terrestrial vertebrates

Received: June 1, 2021; Accepted: June 11, 2021.

(c) The Author(s). 2021 This is an Open Access article distributed under the terms of the Creative Commons License (http://creativecommons.org/licenses/by/4.0/) which permits unrestricted use, distribution, and reproduction in any medium or format, provided the original work is properly cited. and at least 51,000 species of insects identified already (1). This species richness definitely is still underestimated as the rate of new and cryptic species discovery remains high. However, China's genetic resources have also decreased sharply in the past decades due to its large human population and intensive human activities (2). Nearly half of China's animals are found nowhere else and many are archaic and distinctive evolutionary lines nowadays at serious risk of extinction, such as the Giant panda (Ailuropoda melanoleuca) and Chinese alligator (Alligator sinensis) (3). Consequently, the high rates of 
species discovery and loss have led to the urgent need for standardized methods to assess varied animal groups.

Conventional taxonomic approaches for classification of species or breeds mainly rely on the characterization of morphological features, which are time-consuming and in some cases even lead to the disappearance of a species before its description (4). Moreover, both genetic and environmental factors underlie morphological variations. How genetic and environmental factors influence morphological characteristics remains a fundamental question under biological investigations (5). Observations based on morphology thereby are often unclear and challenging for species discovery and delimitation. Although whole genome sequencing and mass spectrometry-based protein profiling have also emerged as high throughput techniques allowing more precise species and breeds assessment $(6,7)$, the tedious analysis and high price slow their development in the field and, as a result, faster and easier alternatives with low costs are preferred.

DNA barcoding is a molecular tool employing sequence divergence in short and standardized gene regions to aid identification and discovery of species (8). It seeks to adopt one or few DNA fragments to efficiently and effectively assign any biological sample to its species regardless of the visual identification of the sample (9). The core idea is based on the fact that certain pieces of DNA, when aligned, can be found to vary merely to a limited degree within species while this variation is much less than between species (10). Therefore, whether samples of diverse species can be differentiated largely depends on the choice of the DNA sequence, which should be easy for amplification using universal PCR primers. Regions from mitochondrial genes usually form barcodes for members of animal kingdom. This is because each mitochondrion possesses insufficient DNA repair mechanisms and multiple copies of naked DNA without the protection of histone proteins, resulting in a 10 -fold higher rate of nucleotide substitution in comparison to nuclear genome $(11,12)$. The accumulation of mutation in mitochondrial DNAs (mtDNAs) helps introduce more sequence diversity to establish phylogenetic relationships among animals and increases the chance to distinguish between closely related species (13).

A short fragment of $\sim 648$ base pairs (bp) at the 5 ' end of the mitochondrial gene encoding the cytochrome $\mathrm{c}$ oxidase subunit 1 ( $C O I)$ enzyme is the first and so far the most broadly used molecular marker for barcoding animals (14). It was reported that more than $95 \%$ of species in test assemblages of different animal groups, mainly insects, birds and fishes, showed characteristic COI sequences after successfully amplified using a universal pair of primers (15). More studies, however, challenged the degree of universality for $C O I$ and its primers for a number of reasons. For instance, the high variability of nucleotide sequences at the $C O I$ priming sites hinders its application to a broader spectrum of animal species (16). To address this issue, selected COI region and primers have been modified for barcoding species like amphibians (17). Yet how well the modifications work for bio-identification of other animals is still questionable, especially when coming to some distinctive and rare lines. As an alternative candidate, mitochondrial $16 \mathrm{~S}$ ribosomal $R N A$ gene is also often used, but its usage is substantially restricted to simple taxonomic analyses of microbiota (18). This is because of the prevalence of insertions and deletions in the non-coding RNA, which is thought to greatly complicate sequence alignments, although successes that $16 \mathrm{~S}$ is superior to $\mathrm{COI}$ are also realized recently for Arthropoda and Amphibians (13, 19, $20)$. In spite of this, whether $16 S$ could supply a sufficient resolution and robustness to discover entire animal kingdom has not been fully explored.

To date, little is known about the effectiveness of DNA barcode for evaluating taxonomic and phylogenetic structures of rare indigenous animals. In the paper present here, we select 54 representative species of distinctive terrestrial animals with 395 samples collected in 15 provinces throughout China, and systematically test the recovery of sequence information with universal primer sets that target short segments of the $C O I$ and $16 S$ barcode regions. The goal of this work focuses on the prospect for investigating the genetic variability of threatened species using barcode sequences of COI and $16 S$ through both distance-based and tree-based approaches. Our efforts will assist policy makers to understand the global patterns of biodiversity and persuade them to develop management strategies for prioritization and hot spots for conservation.

\section{Materials and Methods Sample acquisition}

Animal samples including blood, semen, hair, tissues and faeces were collected in P. R. China following Animal Use Protocols approved by the Animal Care and Ethics Committee of Nanjing Agricultural University. Geographical distribution map of sample collection sites was created by making use of HyperText Markup Language 5 (HTML 5) and JavaScript scripting language (Figure 1). Blood samples were preserved with EDTA, whereas semen and faecal samples were frozen in liquid nitrogen. Hairs and tissues were kept in 75\% ethanol. Species identity was based on morphological characters determined in the field. A total of 395 specimens representing 54 animal species, including 14 indigenous breeds of farm animals (4 species) in China, were used in this study (Supplemental Table S1). Some species were rare and represented by a single specimen solely, but for the majority multiple specimens were analyzed.

\section{DNA extraction}

DNA from blood samples was extracted using TINAamp Blood DNA Kit (DP348-03), while TINAamp Genomic DNA Kit (DP304-02) was adopted for DNA extraction from tissues. DNA from animal semen was purified using 


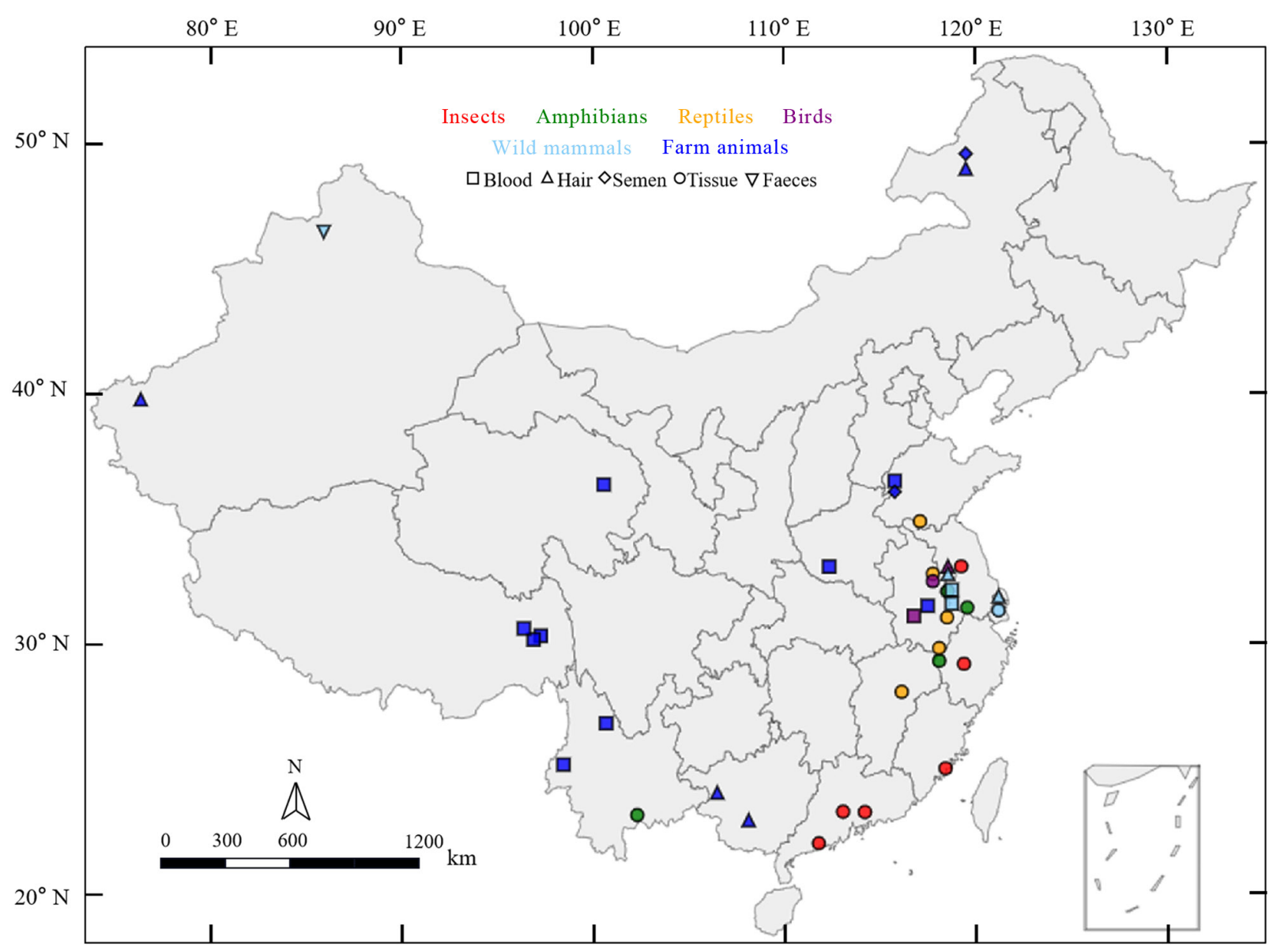

Figure 1. Map of China with sampling sites indicated according to the classification and properties of the specimens. Detailed sample information is shown in Table S1.

Omega Forensic DNA Kit (D3591-02), and Omega Stool DNA Kit (D4015-01) was applied for camel faecal samples. All procedures were carried out according to the manufacturer's protocol. DNA concentration and purity were assessed by Thermo Fisher Scientific NanoDrop One. DNA from animal hairs was extracted using alkaline lysis method as mentioned in (21). In brief, hair follicles from 10 hairs were boiled in $50 \mu \mathrm{L} 0.2 \mathrm{M} \mathrm{NaOH}$ for 15 min, and $50 \mu \mathrm{L}$ Tris- $\mathrm{HCl}(\mathrm{pH}=6.0)$ was then added before following experimental performance.

\section{$\mathrm{COI}$ and $16 S$ amplification}

Less than $150 \mathrm{ng}$ DNA or $5 \mu \mathrm{L}$ hair lysate was used as template to amplify mitochondrial $\mathrm{COI}$ and $16 \mathrm{~S}$ fragments. PCR was carried out in a $50 \mu \mathrm{L}$ volume reaction using a Taq DNA polymerase (Vazyme, P213) with proofreading activity. Two sets of primers, COI-C0 and $\mathrm{Chm} 4$ (Table 1), were used depending on species to

Table 1. Sequence information of universal PCR primers for $\mathrm{COI}$ and $16 S$.

Base pairs underlined indicate 2-fold degenerate bases.

\begin{tabular}{|c|c|c|c|c|}
\hline Barcode & Primer & Name & Primer sequence 5'--3' & Source \\
\hline \multirow[t]{4}{*}{$\mathrm{COI}$} & $\mathrm{COI}-\mathrm{C} 0$ & COI-C02 & AYTCAACAAATCATAAAGATATTGG & [1] \\
\hline & & COI-C04 & ACㅍTCRGGRTGACCAAAAAATCA & \\
\hline & $\mathrm{Chm} 4$ & Chmf4 & TYYTCWACWAA $\underline{Y} C A \underline{Y} A A A G A \underline{Y A T C G G}$ & [1] \\
\hline & & Chmr4 & 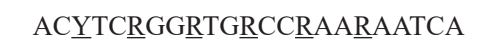 & \\
\hline \multirow[t]{2}{*}{$16 S$} & $16 \mathrm{~S}$ & 16Sar-L & CGCCTGTTTATCAAAAACAT & [2] \\
\hline & & $16 \mathrm{Sbr}-\mathrm{H}$ & CCGGTCTGAACTCAGATCACGT & \\
\hline
\end{tabular}

[1] CHE, J., CHEN, H. M., YANG, J. X., JIN, J. Q., JIANG, K., YUAN, Z. Y., MURPHY, R. W. \& ZHANG, Y. P. 2012. Universal COI primers for DNA barcoding amphibians. Mol Ecol Resour, 12, 247-58.

[2] PALUMBI, S. J. D. O. Z. \& HAWAII, K. M. L. U. O. 1991. Simple fool's guide to PCR. Dept of Zoology \& Kewalo Marine Laboratory University of Hawaii. 
amplify the same barcoding region of $\mathrm{COI}$ (17). $16 \mathrm{~S}$ barcoding region was amplified with $16 \mathrm{Sar}-\mathrm{L}$ and $16 \mathrm{Sbr}-\mathrm{H}$ primers (Table 1) by using $1.2 \mu \mathrm{M}$ of each for all species (22). PCR products were visualized on $1 \%(\mathrm{w} / \mathrm{v})$ agarose gels with ethidium bromide and recovered using TIANgel Midi Purification Kit (DP209). Details of PCR conditions and products for each sample are shown in Supplemental Table S2.

\section{TA cloning}

Purified PCR products were linked to pMD19-T vector with pMD ${ }^{\text {тм}} 19-\mathrm{T}$ Vector Cloning Kit (TaKaRa 6013) in a $10 \mu \mathrm{L}$ reaction containing $4.75 \mu \mathrm{L}$ PCR product, $0.25 \mu \mathrm{L}$ vector and $5 \mu \mathrm{L}$ Solution I. The mixture was incubated at $16{ }^{\circ} \mathrm{C}$ for $30 \mathrm{~min}$ and then transformed into DH5 $\alpha$ competent cells (Vazyme, C502) according to the instruction. After gentle mixing, competent cells were placed on ice for $30 \mathrm{~min}$, and then in $42{ }^{\circ} \mathrm{C}$ water bath for $45 \mathrm{~s}$ before quick transfer into ice for $3 \mathrm{~min}$. Bacteria were cultivated in $100 \mu \mathrm{L} \mathrm{LB}$ media at $37^{\circ} \mathrm{C}$ for $10 \mathrm{~min}$ and spread on LB agar plates containing $0.1 \mathrm{mg} / \mathrm{mL}$ ampicillin, $0.04 \mathrm{mg} / \mathrm{mL} \mathrm{X}$-gal and $25 \mu \mathrm{g} / \mathrm{mL}$ IPTG for selection. Single white colonies were picked up and cultivated for sequencing.

\section{Sanger sequencing}

DNA barcoding regions were sequenced on both directions using PCR primers directly for PCR products, or using RV-M: GAGCGGATAACAATTTCACACAGG and M13F-47: CGCCAGGGTTTTCCCAGTCACGAC primers for TA clonings. The number of colonies sequenced is in line with one colony per $50 \mathrm{bp}$ of barcoding region unless identical barcode sequence was sequenced twice. Sequencing results were assembled, aligned and annotated using DNASTAR Lasergene package before manually checked. All gained sequences were confirmed by sequence similarity search available in GenBank and deposited under the following accession numbers: MZ046006-MZ046047, MZ046083-MZ046118, MZ046726-MZ046731, MZ047098-MZ047179, MZ048967-MZ049527, MZ050069-MZ050116, MZ050118-MZ050213, MZ050493-MZ050515, MZ061667-MZ061700, MZ068220-MZ068223, MZ098871-MZ099441, MZ099449-MZ099455 for COI sequences; MZ031856-MZ031915, MZ040165MZ040224, MZ040226-MZ040319, MZ040322MZ040403, MZ040406-MZ040487, MZ040500MZ040596, MZ040600-MZ040754, MZ040922MZ041011, MZ041035-MZ041093, MZ041115MZ041206, MZ042146-MZ042231, MZ042371MZ042467, MZ042537-MZ042621, MZ042714MZ042791, MZ042801 and MZ061594-MZ061630 for $16 S$ sequences.

\section{Sequence diversity}

DNA Sequencing Polymorphism (DnaSP) was adopted to screen for haplotypes and polymorphic sites by moving a 200-bp-long sliding window $1 \mathrm{bp}$ at a time, and to calculate haplotype diversity (Hd), nucleotide diversity $(\pi)$ and the average number of nucleotide differences $(\mathrm{K})$. Parameters regarding the length and composition of DNA and protein sequences were determined by MEGA

$\mathrm{X}$. Translations of $\mathrm{COI}$ haplotypes were checked via EditSeq with codon usage and isoelectric point calculated automatically using genetic codes for mitochondrial DNA.

\section{Distance and Tress matrices}

DNA or protein sequences were aligned by the Clustal $\mathrm{W}$ method before the following bioinformatic calculations contained in the software package of MEGA X. Inter/ intraspecific genetic distance for $C O I$ and 165 was plotted using the $\mathrm{R}$ programming language based on Kimura's 2-parameter (K2P) model. K2P is one of the optimal theories for small pairwise distances, and is widely used to calculate sequence divergences in DNA barcoding literatures despite it is criticized (23). For each animal class, the minimum interspecific distance was compared with the maximum intraspecific distance in order to determine the presence or absence of a barcoding gap. Neighbor joining and maximum likelihood trees construction were done using the "pairwise deletion" option for the treatment of gaps and missing data. Node support was computed by 1000 bootstrap replicates with K2P distance for DNA data and Jones-Taylor-Thornton (JTT) algorithms for proteins as a model of substitution. Phylogenetic dendrograms were finally exported into the web based iTOL (https://itol.embl.de/itol.cgi) tool for annotation and visualization.

\section{Statistical analysis}

All data are expressed as the average \pm SD. Spearman's correlation coefficient was used on data processing to assess the relationship between the variables. Data analysis was performed using the IBM SPSS software. Comparisons between two groups were made using Student's t-test. The level of significance was set at $\mathrm{p}<$ 0.05 .

\section{Results and Discussion \\ Isolation and characterization of COI DNA barcodes}

Both COI-C0 and Chm4 primer sets were reported to span the same positions in the COI gene of amphibians (17). The Chm4 primer set was designed to have more 2-fold degenerate bases in comparison to COI-C0 (Table 1). Therefore, the Chm4 primer set is utilized in this study only when the COI-C0 primers fail to amplify a perfect PCR product. In this experimental setting, 1480 COI sequences recovered from 391 specimens ( $>98.98 \%$ success) yield 787 unique haplotypes ranging from 616 to $663 \mathrm{bp}$ with an average of $652 \mathrm{bp}$, among which 592 haplotypes are defined from 1094 sequences generated by COI-C0 for $28 / 6$ species/breeds, while the rest are from 25/8 species/breeds through Chm4 primer pair (Figure 2a, Supplemental Table S2 COI 1st pair and Table S3). Notably, haplotype sequences of $C O I$ are only shared 

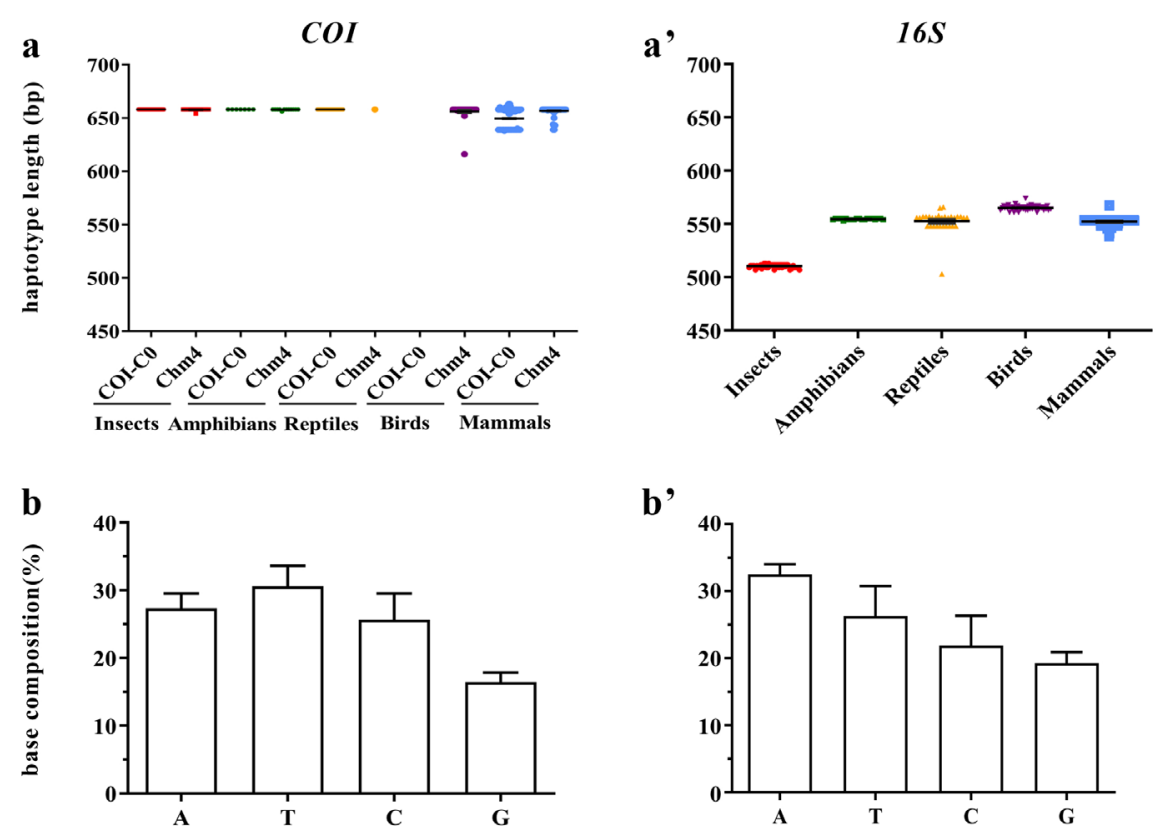

b'
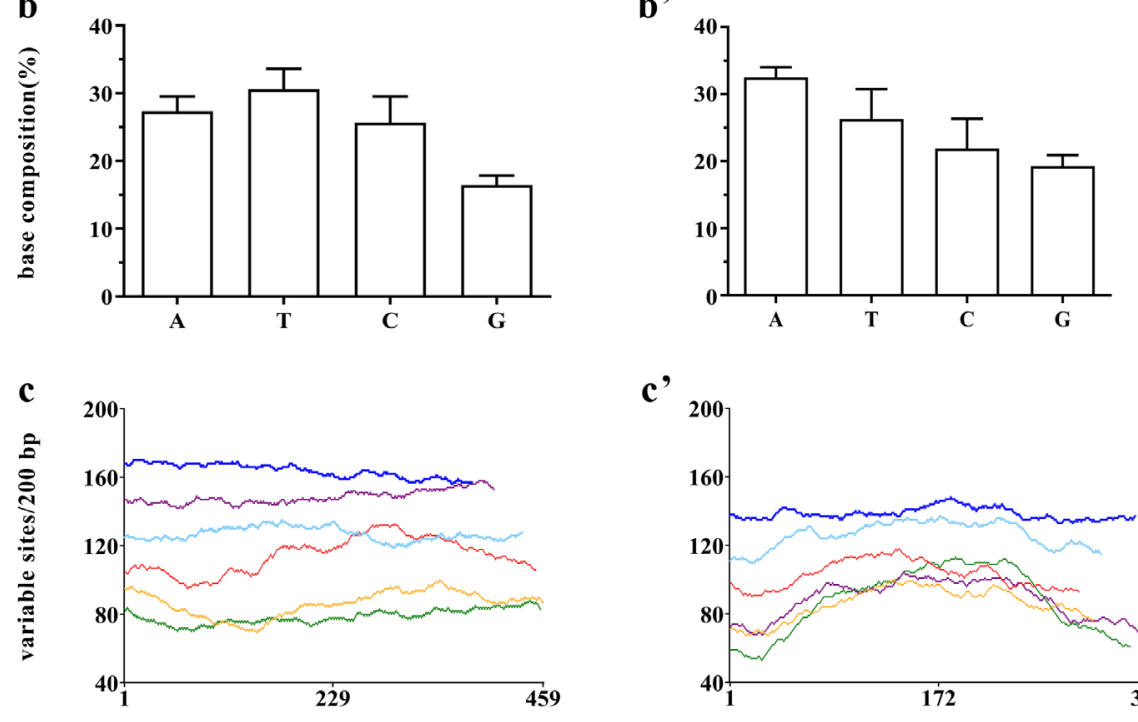

c'

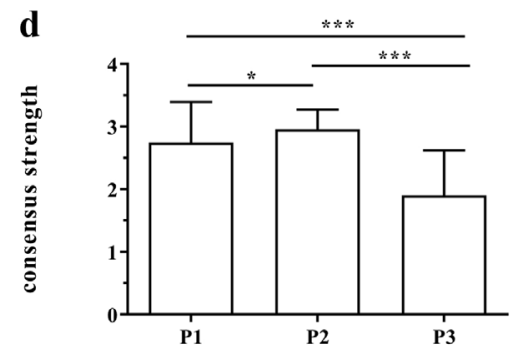

$\mathbf{d}^{\prime}$

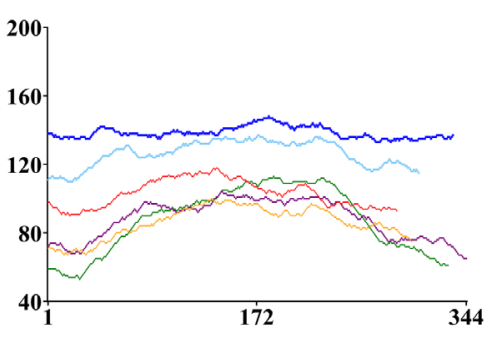

Birds $\rightarrow$ Wild mammals _- Farm animals

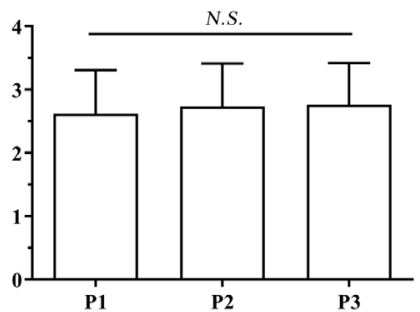

Figure 2. Isolation and characterization of $\mathrm{COI}$ and $16 \mathrm{~S}$ barcodes. (a-b') Sequence length and average nucleotide composition of $\mathrm{COI}\left(\mathrm{a}\right.$ and $\mathrm{b}$ ) and 16S (a' and b') haplotypes. ( $\mathrm{a}$ and $\mathrm{a}^{\prime}$ ) Each dot represents a unique haplotype. (c and c') Sliding window graph comparing the number of variable sites for $\mathrm{COI}$ and $16 \mathrm{~S}$ haplotypes. Each window size of $200 \mathrm{bp}$ is slid through the full segment 1 $\mathrm{bp}$ at a time, resulting in 459 windows for $\mathrm{COI}$ (c) and 344 for $16 \mathrm{~S}$ (c'). Animal groups are indicated by lines with different colors. (d) Variation of the triplet code of $\mathrm{CO}$ / haplotypes. Consensus strength is scored according to the Alignment Report given by MegAlign using Lasergene software package and plotted according to the positions of genetic code. $\left({ }^{*}\right) p<0.05 ;\left({ }^{* * *}\right) p<0.001$. The same rules are applied to non-coding $16 \mathrm{~S}$ haplotypes but the codon positions are defined randomly (d'). (N.S.) not significant.

among various breeds of farm animals, but not at the level of species.

The average nucleotide composition of $C O I$ haplotypes is $27.32 \%$ A, 30.58\% T, 25.65\% C and 16.45\% G (Figure 2b). The GC content of these sequences varies from $25.60 \%$ to $52.40 \%$, and identifies insects having the lowest GC content, consistent with what was reported before (24). As shown in Figure 2c, the density of variable sites in $200 \mathrm{bp}$ windows along the alignment of COI haplotypes does not fluctuate widely for each animal group. Even so, the third position of genetic code generally is the least conserved and the second codon position shows slightly smaller variation than the first position (Figure 2d), in line with the "wobble phenomenon" during protein translation (25). However, this is not the case for $16 \mathrm{~S}$, which is a noncoding RNA and has all positions varying at an identical rate (Figure 2d').

\section{Isolation and characterization of $16 S$ DNA barcodes}

The $16 \mathrm{~S}$ primers manage to obtain 1255 sequences from 390 out of 395 collected samples comprising 53/14 species/breeds in this study (Supplemental Table S2). 

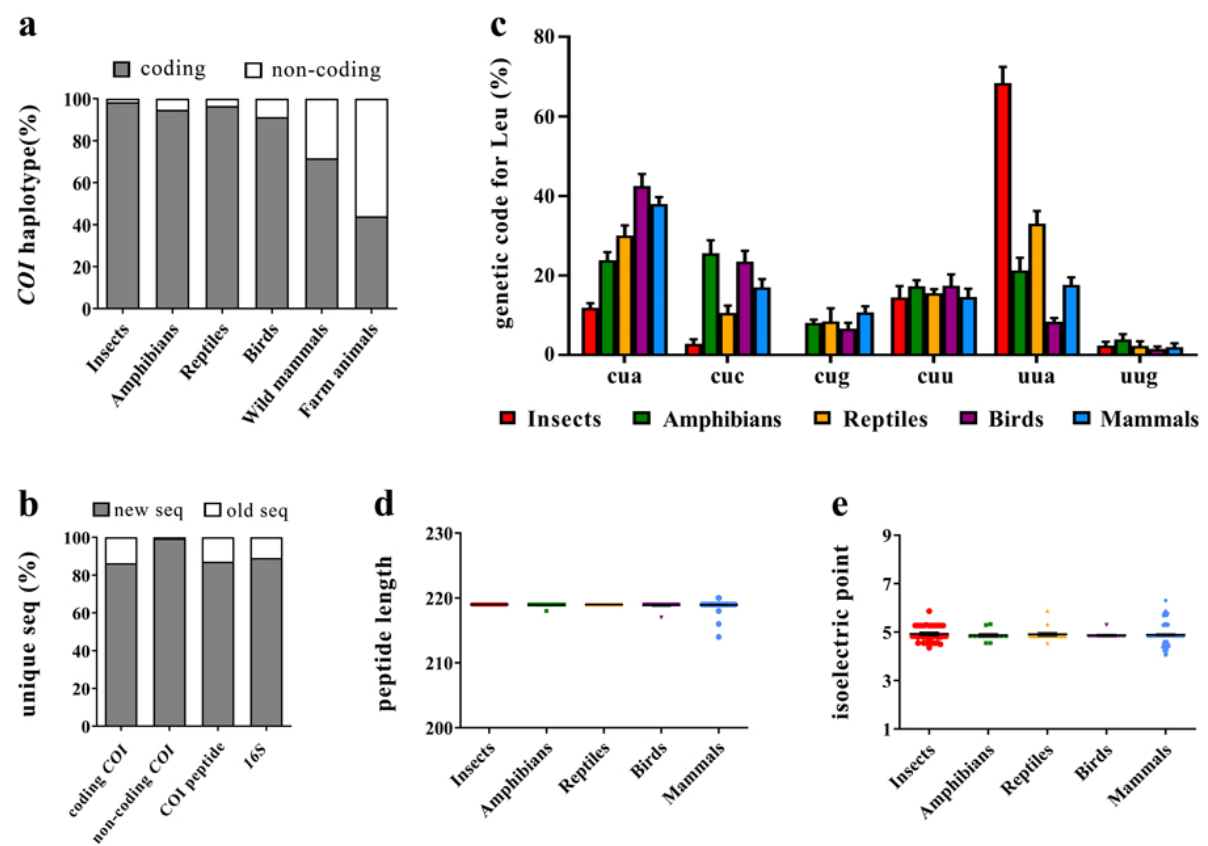

Figure 3. Isolation and characterization of $\mathrm{COI}$ peptides. (a) Translation of $\mathrm{CO}$ / haplotypes using genetic codes for mitochondrial DNA among different animal groups as indicated. (b) Percentage of novel sequences (seq) identified after BLAST similarity search in Genbank. (c) Usage of synonymous codons for leucine in COI peptides. Animal classes are indicated by various colors. (d and e) Sequence length and isoelectric point of $\mathrm{COI}$ peptides. Each dot represents a unique protein sequence.

After removal of identical sequences within any one species, the barcode library is reduced to 592 unique haplotype segments with an average of $550 \mathrm{bp}$ and lengths ranged from 503 to 574 bp (Figure 2a' and Supplemental Table S4). Of these, $89.02 \%$ haplotypes are newly discovered in current study after aligned to the $16 \mathrm{~S}$ sequences in GenBank database. Similar to $\mathrm{COI}$ barcodes, there is no haplotype sequence of $16 \mathrm{~S}$ found to be shared between species either.

The base compositions of $16 \mathrm{~S}$ are always biased towards $\mathrm{A}$ and $\mathrm{T}$, which together are present in a higher proportion than GC, while the latter varies from $20.70 \%$ to $49.38 \%$ (Figure 2b'). As usual, insects have the lowest GC content. Molecular diversity indices of both $\mathrm{COI}$ and $16 \mathrm{~S}$ barcodes are given in Table 2 for comparison. Overall, $16 S$ shows lower nucleotides diversities $(\pi)$ as well as average number of nucleotide differences $(\mathrm{K})$ for all animal groups when compared with $C O I$. Also distinct from $C O I, 16 S$ haplotypes seem less polymorphic in their initial and final portions for all animal groups except for farm animals, which have the largest amount of variable sites owing to having too many $16 \mathrm{~S}$ haplotypes for test (Figure 2c').

\section{Isolation and characterization of COI protein peptides}

Translation of COI haplotypes is also examined as $C O I$ is a protein-coding gene with essential function for oxidative phosphorylation (26). Results reveal that out of 787 unique COI barcodes, only 462 consisting of 51/11 species/breeds are functional domains containing no stop codon (Supplemental Table S3). Of those nonsense mutant fragments, more than $99 \%$ are novel sequences, majority of which are derived from mammalian samples (wild mammals and farm animals) (Figure $3 \mathrm{a}$ and $\mathrm{b}$ ). In this respect, the percentage of new COI haplotypes able to encode proteins is $>13 \%$ lower $(86.36 \%)$, close to that of $16 \mathrm{~S}(89.02 \%)$ when aligned to the data in GenBank. The 462 functional COI barcodes mentioned above in total isolate 318 unique peptides, for all of which Leucine is the amino acid used most frequently although obvious codon bias for Leucine is observed among animal groups (Supplemental Table S5 and Figure 3c). The average length of the $318 \mathrm{COI}$ peptides is 219 amino acids, within a narrow range from 214 to 220 amino acids, correlated with the small window of their isoelectric points between 4.059 and 6.308 (Figure 3d and e). When compared to data available in Genbank, around $87.11 \%$ peptides are characterized to be novel COI sequences (Figure 3b). However, sharing of peptide sequence is detected between species of not closely related birds or mammals, indicating COI proteins, in contrast to $C O I$ nucleotides, possess higher conservatism in the course of species evolution (Supplemental Table S5).

\section{Barcoding gap and Species resolution}

The primary application of barcode markers is to discriminate species. Methodological approaches for species delineation using DNA barcodes are commonly dependent on genetic distance-based measures, which rely on the assumption of a remarkable separation between intraspecific variation and interspecific divergence in the selected marker, also referred to as the barcoding gap $(27,28)$. K2P analysis thus is conducted using COI haplotypes, coding $\mathrm{COI}$ and $16 \mathrm{~S}$ haplotypes to calculate 

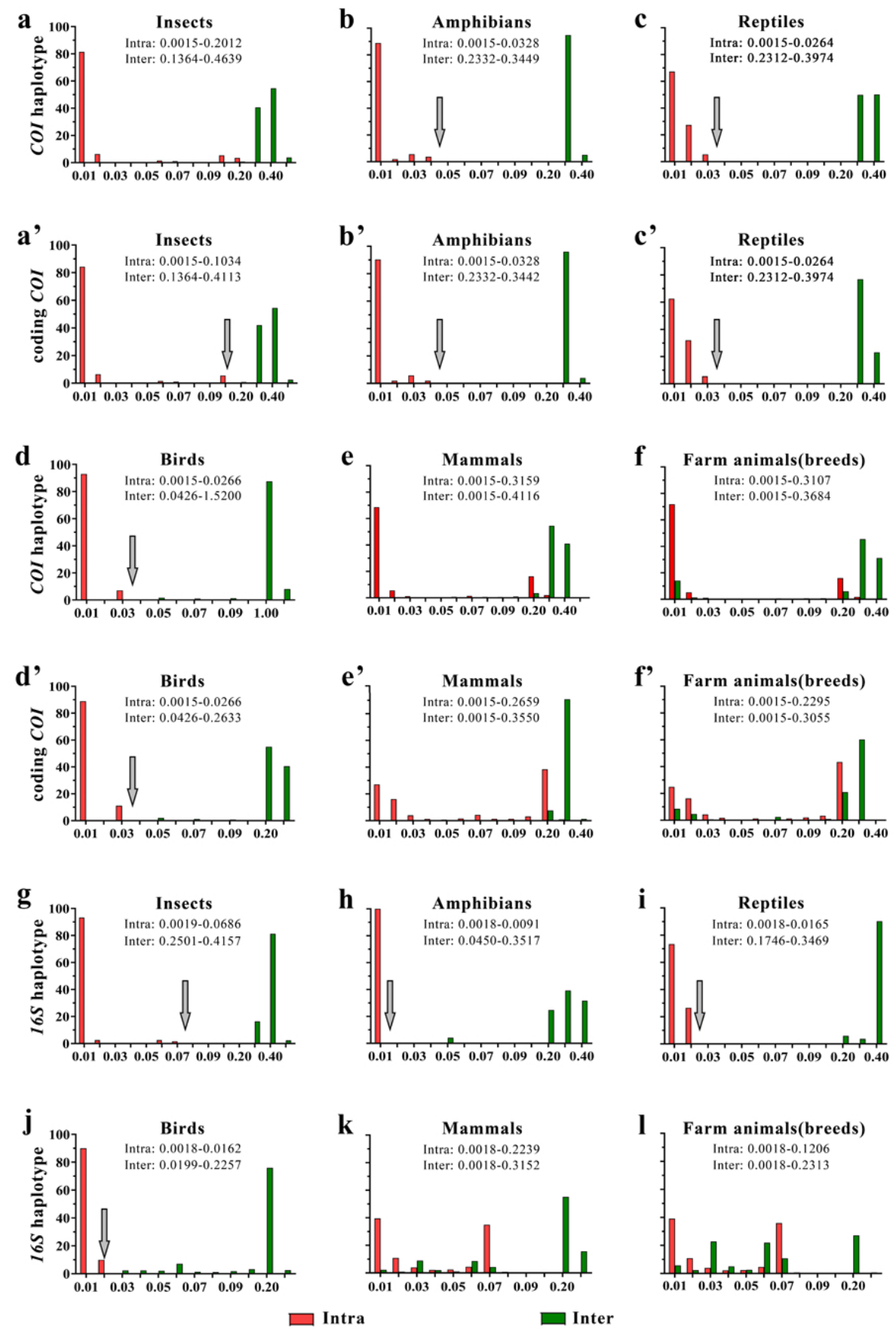

Figure 4. Histograms displaying the intraspecific and interspecific K2P pairwise sequence divergences for all $\mathrm{CO}$ haplotypes (a-e), coding COI (a'-e') and all 16S haplotypes (g-k) of animal classes as indicated. Genetic distances are also compared within and between the 14 breeds of farm animals with COI haplotypes (f), coding COI (f') and $16 S$ (I) haplotypes. The minimum and maximum inter/intraspecific distance is depicted for each comparison. Gray arrows indicate the existence of barcoding gaps.

genetic distance respectively. The two COI datasets give comparable outcomes among all animal groups except for insects, in which coding COI is moderately better, displaying a murky gap with the minimum interspecific distance higher than the maximum intraspecific distance (Figure 4a-f'). Additionally both translatable COI and $16 \mathrm{~S}$ haplotypes exhibit barcoding gaps for insects, amphibian, reptiles and birds, even though COI protein peptides are shared between some birds (Figure 4 and Supplemental Table S5). Nevertheless, both fail to show a separate distribution for mammals within and between species or breeds (Figure 4e-f').

Sequence datasets then are further evaluated using BLAST searches, which typically employ distance-based algorithms for pairwise alignments to assess species resolution (6). Performance of BLAST using COI peptides no surprise deliver the lowest species resolution because of the highest degree of sequence conservation (Figure 5a and Supplemental Table S5). As for DNA barcodes, COI overall offers better species resolution than $16 S$ (Figure 5a), 
Table 2. Genetic diversity of $\mathrm{COI}$ and $16 \mathrm{~S}$ barcodes generated in the present study.

\begin{tabular}{cccccccc}
\hline Species & barcode & N & S & H & Hd (SD) & $\pi$ (SD) & K \\
\hline Insects & COI & 138 & 363 & 62 & $0.9240(0.0120)$ & $0.19422(0.00208)$ & 126.246 \\
& $16 S$ & 122 & 247 & 51 & $0.9010(0.0160)$ & $0.18603(0.00507)$ & 90.412 \\
Amphibians & COI & 37 & 261 & 19 & $0.9350(0.0220)$ & $0.16619(0.01298)$ & 108.853 \\
& $16 S$ & 42 & 194 & 20 & $0.9360(0.0180)$ & $0.15242(0.00657)$ & 80.632 \\
Reptiles & COI & 78 & 308 & 35 & $0.9050(0.0210)$ & $0.15734(0.00861)$ & 103.528 \\
& $16 S$ & 69 & 213 & 30 & $0.8880(0.0270)$ & $0.14312(0.00722)$ & 70.987 \\
Birds & COI & 54 & 448 & 26 & $0.9590(0.0100)$ & $0.18004(0.02340)$ & 108.92 \\
& $16 S$ & 69 & 213 & 44 & $0.9740(0.0090)$ & $0.09766(0.00357)$ & 53.030 \\
Wild Mammals & COI & 270 & 405 & 157 & $0.9847(0.0028)$ & $0.20079(0.00152)$ & 127.703 \\
& $16 S$ & 236 & 297 & 121 & $0.9775(0.0038)$ & $0.12394(0.00193)$ & 62.465 \\
Farm animals & COI & 903 & 474 & 488 & $0.9610(0.0041)$ & $0.14981(0.00229)$ & 86.592 \\
& $16 S$ & 717 & 368 & 326 & $0.9666(0.0027)$ & $0.05204(0.00205)$ & 27.684 \\
\hline
\end{tabular}

$\mathrm{N}=$ number of sequences; $\mathrm{S}=$ number of polymorphic sites; $\mathrm{H}=$ number of haplotypes; $\mathrm{Hd}$ (SD)=haplotype diversity (standard deviation); $\pi$ $(S D)=$ nucleotide diversity (standard deviation); K=average number of nucleotide differences.

especially for insects, amphibians, reptiles and wild mammals (Figure 5b). Although the capability to make species-level identifications diverges tremendously for both DNA markers, a statistically significant correlation $($ rho $=0.76, \mathrm{p}<0.01)$ is noted between the average resolutions of $C O I$ and $16 \mathrm{~S}$ for each species or breed of farm animals (Figure 5c, Supplemental Table S3 and S4). In addition, BLAST results give a general impression that translatable COI barcodes have higher levels of species discrimination than their non-coding counterparts (Figure 5a). However, this is not always true since for species or breeds such as Golden monkey (Rhinopithecus roxellanae), Tibetan cattle (Bos grunniens), Sanhe horse and Tibetan horse (Equus caballus), some non-functional counterparts can perform even better (Supplemental Table S3).

\section{Phylogenetic relationships}

Contrary to divergence-based barcoding gap, construction of evolutionary trees places emphasis on conservation areas which are designed or prioritized according to their phylogenetic diversity (29). The most popular approaches to reconstruct phylogenies are the neighborjoining (NJ) and the maximum likelihood (ML) algorithm. Although both phylogram constructions could correctly discriminate most COI haplotypes, with exception discussed below, NJ turns out to act better in light that it allocates related species closer to each other as hinted by the line colors representing animal classes in Figure 6. Yet the topologies of ML tree search sometimes can be more similar to the traditional taxonomic classifications. For example, rather than directly rooted from the same node as Sika deer (Cervus nippon) (Figure 6a), a

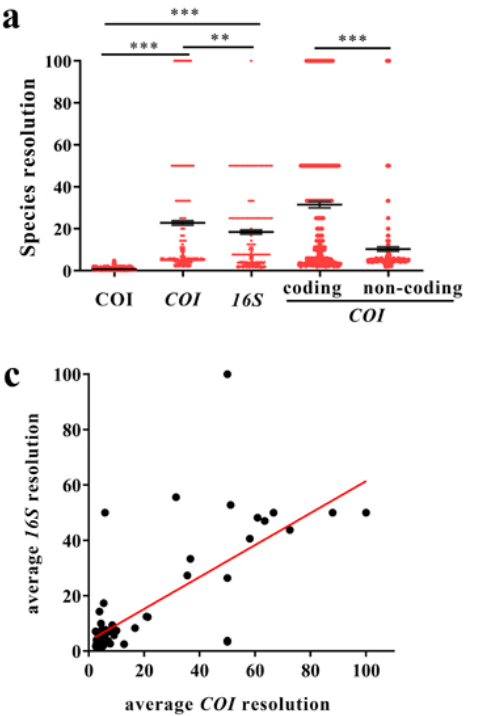

b

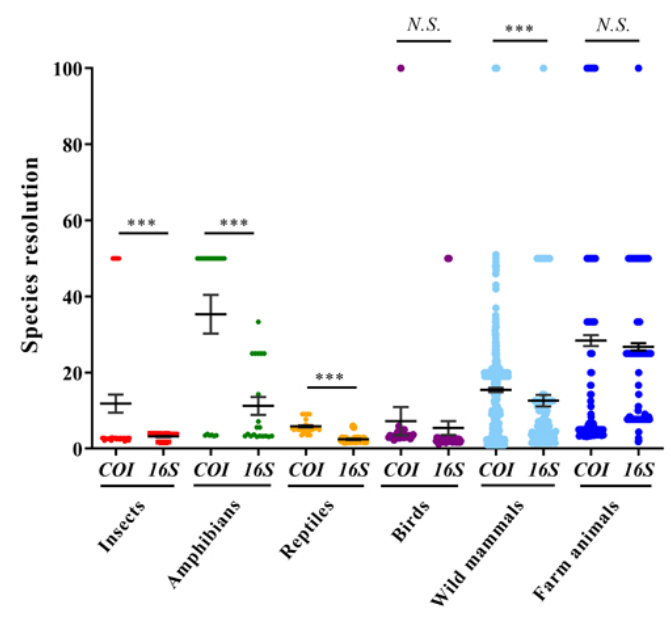

Figure 5. Species resolution provided by $\mathrm{COI}, 16 S$ DNA barcodes and COI peptides (a) for different animal groups (b) as indicated. Species resolution of unique sequences is predicted using BLAST searches based on reference libraries in Genbank, where reference sequences might be deposited unequally for various species. $\left.\left({ }^{* *}\right) p<0.01 ;{ }^{* * *}\right) p<0.001$; (N.S.) not significant. (c) Correlation between the average resolutions of $\mathrm{COI}$ and $16 \mathrm{~S}$ for each species or breed of farm animals. Each dot is plotted according to the average values of resolution from all $\mathrm{COI}$ or $16 \mathrm{~S}$ haplotypes belonging to the same species or breed. Spearman's correlation coefficient rho=0.76 $(p<0.01)$. 
$\mathbf{a}$
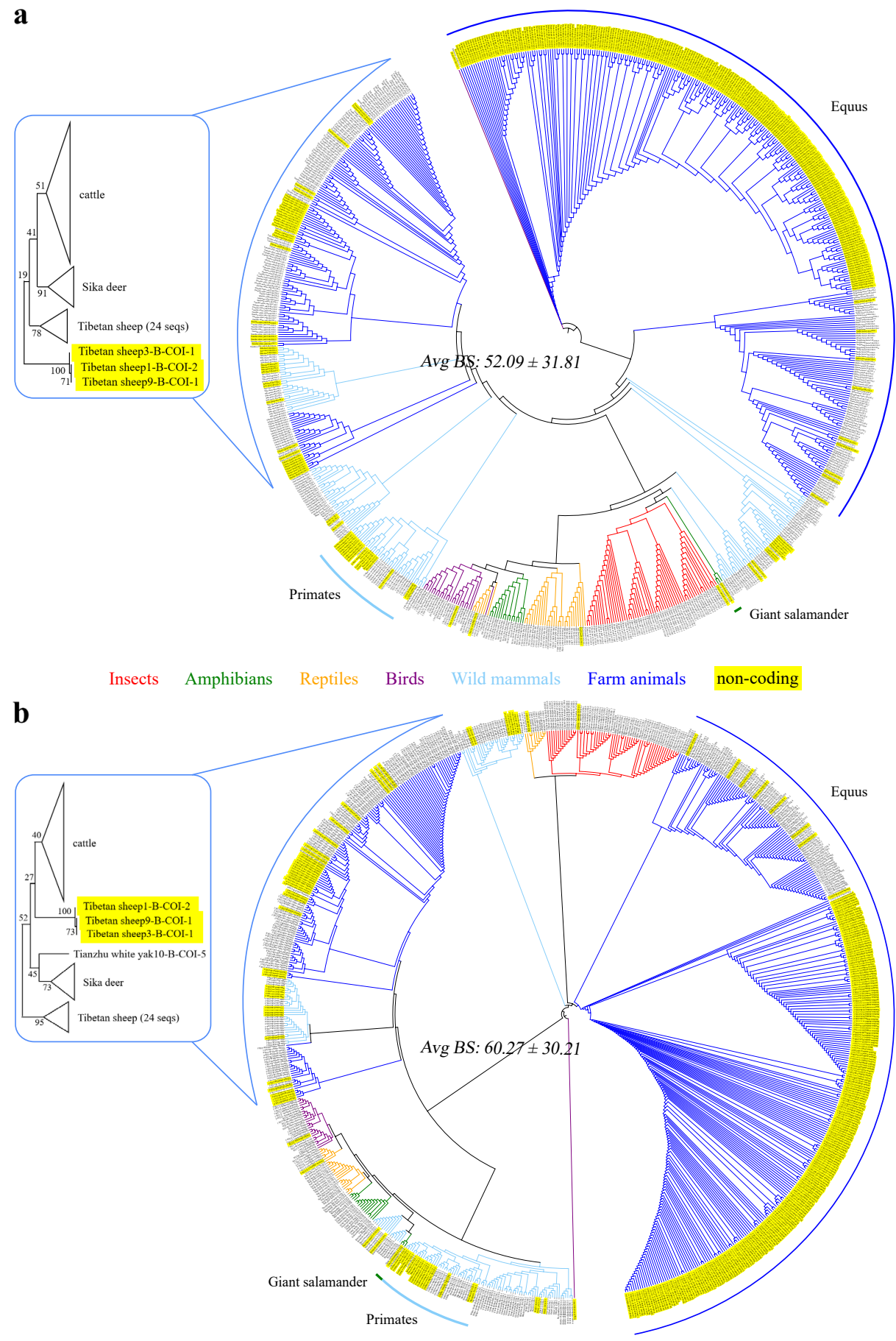

Figure 6. NJ (a) and ML (b) trees inferred from COI haplotypes using K2P distances. Highlighted boxes show a more detailed view of Sika deer, Tibetan sheep and cattle with bootstrap values. Sequences from Giant salamander, primates and Equus (horse and donkey) are also highlighted. (Avg BS) average bootstrap support.

COI coming from cattle gather together and form a parallel branch with sheep before grouping with the branch containing deer according to the ML inference (Figure 6b). Unexpectedly, the Tibetan sheep (Ovis aries) haplotypes here are sequences bearing stop codons and considered as outgroup from the other 24 COI barcodes of sheep, whereas one coding sequence from Tianzhu white yak (cattle) is still misplaced with deer (Figure 6b box), highlighting a stronger discriminatory power of nonsense mutants that is usually ignored as reviewed by earlier studies $(30,31)$. Exceptional case common for both tree-building methods is Giant salamander (Andrias davidianus), whose coding peptides also fail to cluster with other Amphibians in protein phylogenies based on the JTT models (Supplemental Figure S1), indicating a special position of the archaic species in the evolutionary history (32).

It is noticeable that the NJ approach is much more superior to $\mathrm{ML}$ when it comes to $16 \mathrm{~S}$ haplotypes (Figure 7). The average bootstrap proportion of NJ somehow is a little 

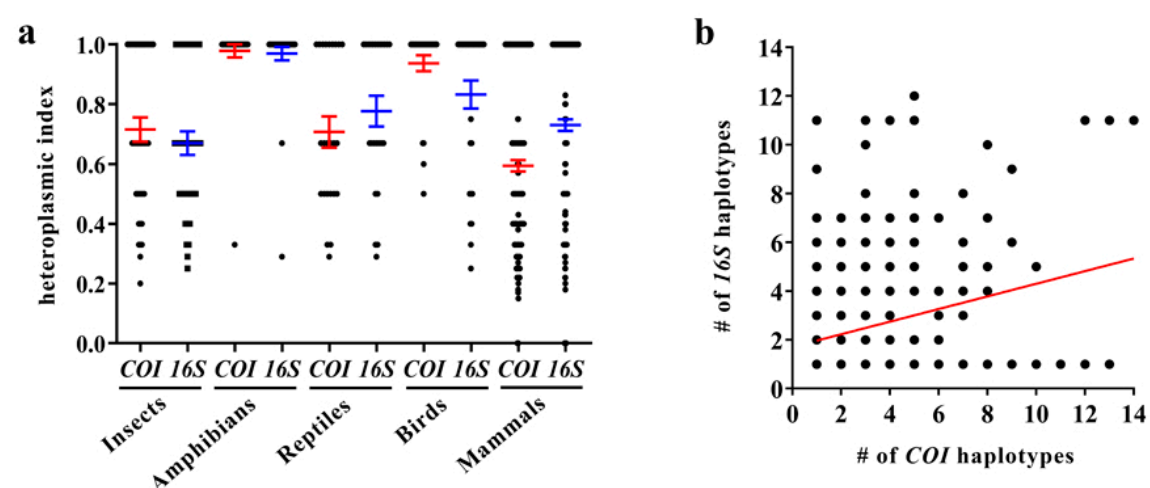

Figure 8. Heteroplasmic conditions in mitochondrial COI and 16S markers. COI and 16S are sequenced directly using PCR primers or after TA cloning, from which bacterial colonies are picked up in line with one colony per 50bp DNA unless the same sequence is captured twice. Heteroplasmy then is monitored either by the chance to obtain identical sequence ("heteroplasmic index" in a) or by the copy number of haplotypes generated (b). (b) Correlation between the copy numbers of COI and 16S barcodes for each individual specimen. Spearman's correlation coefficient rho $=0.18(p<0.01)$.

bit lower than ML, but the NJ algorithm identifies all animal classes accurately with each individual $16 S$ nearly perfectly assigned into their right sites. However, some $16 S$ sequences originated from primates as well as Equus (horse and donkey) are found to be mixed within their groups and not arranged according to their species. These failures are also detected in phylogenetic trees constructed by $C O I$, implying they have a very close kinship (Figure 6).

Different from the universal bar code consisting of a series of vertical bars that are printed on commercial products, DNA barcodes are genetic markers seeking to use sequence message of any biological sample, regardless of morphological identification of the sample, to address questions relating to taxonomy, ecology and evolution (33). A considerable amount of studies have evaluated the performance of a variety of barcode markers with respect to both their ease of PCR amplification and their capacity to delineate species (34). By making use of previously reported primers, here we manage to amplify and isolate DNA barcodes from nearly all Chinese terrestrial animals sampled. Totally 787 COI and $59216 S$ unique haplotypes are characterized in the survey of 54/14 animal species/ breeds. Sequences of selected $C O I$ and $16 S$ region are shared inside species but not between species with a low GC content on average, which is a typical feature of mitochondrial genome. In general, COI markers are longer and more polymorphic than $16 S$ as reviewed by parameters of nucleotides diversities $(\pi)$ and average number of nucleotide differences (K) (Table 2). These results clearly demonstrate that both $\mathrm{COI}$ and $16 \mathrm{~S}$ primer pairs possess high degree of universality, competent to capture targeting segments efficiently from distinctive and rare lines of insects, amphibians, reptiles, birds and mammals across China.

In terms of choices of segments for barcoding and species diagnosis, DNA sequences that evolve slowly, like ribosomal genes in nucleus particularly, often do not differ among closely related organisms. Conversely, sequences that evolve rapidly may overwrite the traces of ancient affinities, but regularly reveal divergence between closely related species. Overwhelming evidence has suggested that mitochondrial genome is more likely to supply suitable candidate regions for barcoding animals for its maternal inheritance, non-introns and rapid evolution $(35,36)$. In order to assess the performance of mtDNAs in our context, distance-based and tree-based approaches are utilized to analyze the data libraries of $\mathrm{COI}$ and $16 \mathrm{~S}$ garnered in this study. Substantially, all methods tend to be congruent with regards to success or failure. The opportunities to distinguish species dependent on the two markers are high for insects, amphibians, reptiles and birds with recognizable barcoding gaps, though $C O I$ appears to provide better species resolution after sequence alignment via BLAST, which might be a bias brought in by the reference databases available at this moment in Genbank (28). Furthermore, it will be more challenging to differentiate indigenous mammals using $\mathrm{COI}$ and $16 \mathrm{~S}$ because neither of them is capable to display a perfect species boundary according to our exploration. This might be a reason why until now fewer barcode records of $C O I$ and $16 S$ are accessible for mammals despite their prevalence in amphibians and reptiles. Nevertheless, it should be noted that the phylogenetic pattern reconstructed with $16 \mathrm{~S}$ through $\mathrm{NJ}$ algorithm seems more reliable than $C O I$, providing a picture generally analogous to the conventional taxonomic classifications. In sum, we speculate that both mitochondrial $\mathrm{COI}$ and $16 \mathrm{~S}$ could function comparably at least as an eligible barcode marker for most species involved in current study, and that application of the two genetic markers relying on bioinformatic approaches should be cautious on a caseby-case basis.

In contrast to earlier studies, we realize high frequency of multiple sequences from one single PCR product as well as many $C O I$ haplotypes bearing non-sense mutations in our experimental setting. In fact, the heteroplasmy in mtDNA and the presence of nuclear pseudogenes of mitochondrial origin (numts) have raised many concerns in the field of barcoding (27). It is known that each eukaryotic cell could contain approximately 1000 copies of mtDNA, leading to a condition called heteroplasmy, where both wild-type and mutant mtDNA molecules 
Table 3. COI sequences and haplotypes obtained from the same samples using two pairs of PCR primers.

\begin{tabular}{|c|c|c|c|c|c|c|c|c|}
\hline COI PCR & Sample ID & $\begin{array}{c}\text { Jungle_- } \\
\text { fowl-B }\end{array}$ & $\begin{array}{c}\text { Tibetan_- } \\
\text { donkey3-B }\end{array}$ & $\begin{array}{c}\text { Tibetan_- } \\
\text { donkey5-B }\end{array}$ & $\begin{array}{c}\text { Yunnan_- } \\
\text { donkey8-B }\end{array}$ & $\begin{array}{c}\text { Yunnan_- } \\
\text { donkey9-B }\end{array}$ & $\begin{array}{c}\text { Dezhou_- } \\
\text { donkey4-B }\end{array}$ & $\begin{array}{c}\text { Dezhou_- } \\
\text { donkey52-S }\end{array}$ \\
\hline \multirow{3}{*}{ 1st pair } & sequence \# & 2 & 3 & 6 & 2 & 9 & 1 & 13 \\
\hline & haplotype \# & 1 & 2 & 5 & 1 & 8 & 1 & 12 \\
\hline & translatable haplotype \# & 0 & 0 & 0 & 0 & 0 & 0 & 0 \\
\hline \multirow{3}{*}{ 2nd pair } & sequence \# & 4 & 4 & 2 & 3 & 3 & 6 & 8 \\
\hline & haplotype \# & 3 & 3 & 1 & 2 & 2 & 5 & 7 \\
\hline & translatable haplotype \# & 3 & 2 & 1 & 1 & 2 & 3 & 3 \\
\hline
\end{tabular}

co-exist within the same cell (37). As illustrated by our data, insects, reptiles and mammals possess strong heteroplasmic phenomena in their mitochondrial genome while the mtDNAs of amphibians and birds hold fewer mutations (Figure 8a). We argue this difference is chiefly because of the properties of specimens sampled rather than due to the nature of the species since it has been demonstrated that the number of mtDNAs each mitochondrion carries is in a tissue-specific manner (38, 39). Yet interestingly, our findings unveil that the copy number of $C O I$ haplotypes is not tightly associated with that of $16 S$ from the same specimen (Figure 8b), implying that evolutions of the two mitochondrial fragments are not synchronous, undergoing a relatively independent pattern that might be controlled by their protein coding abilities.

Meanwhile it is very likely that nuclear numts are also co-amplified by using universal $C O I$ and $16 \mathrm{~S}$ primers. Due to the differences in genetic code between mitochondrial and nuclear genomes, numts are documented and recognized as non-functional copies of mtDNA with diverse sizes naturally integrated into the nuclear chromosome through unknown mechanism (40). Once inserted into nucleus, numts decelerate their evolutionary rate and become molecular fossils of mtDNA, which are thought to be indispensable for recovering ancient relationships $(41,42)$. When examining the translation of COI haplotypes, we uncover a big portion of non-coding pseudogenes with extremes that no translatable $C O I$ is identified from species like Jungle fowl (Gallus gallus) and donkeys (Equus asinus). Under such circumstance, it will be more reasonable to believe that these nonfunctional sequences are primarily derived from numts as extraction methods preferring nuclear DNA is conducted before. To ask whether PCR primers could revise this preference, we check the products amplified from another pair of COI primers (Supplemental Table $\mathbf{S 2}$ COI $2^{\text {nd }}$ pair) for Jungle fowl and donkeys. The same criteria are applied to guide TA cloning and Sanger sequencing for Jungle fowl and six donkeys, from which the highest or the lowest copy numbers of haplotype are detected with COI-C0 primers for each of the three breeds (Tibetan, Yunan and Dezhou Donkey). This time, the chance to gain functional sequence is improved, but no correlation of the copy numbers of $\mathrm{COI}$ haplotypes is observed from the two primer pairs, confirming these primers are picking up homologous sequences from distinct gene loci (Table 3 ).
On the other hand, in order to evaluate the potential misidentifications that could be caused by numts sequences, we decide to keep them in the analyses as outlined above. Our results show that coding $C O I$ in most cases allows methodological approaches to achieve more, but the influence of numts on the accuracy of taxonomic description is limited, suggesting that majority numts screened in this study are merely evolved for brief periods of time. Surprisingly, phylogenetic hierarchies deduced from both NJ and ML could position all COI numts to their expected places at the species level except for primates and Equus, which have been discussed earlier. More intriguingly, in-depth analysis in Figure 6 classifies two groups of non-coding numts from Tibetan sheep, among which seven are clustered with functional COI haplotypes whereas the other three are separated as outgroup with a stronger discriminatory power, at least in ML phylogram, than their coding counterparts. Taken together, these observations indicate that it is indeed not easy to characterize the role of numts as it stands for an ongoing evolutionary procession (41). Of course, it certainly is unfair to treat sequences as numts solely based on translation, which is not feasible for $16 \mathrm{~S}$. Yet it should be acceptable considering that the percentages of novel sequences are parallel among translatable $C O I, 16 S$ and COI peptides but not non-coding COI (Figure 3b). In a word, our preliminary findings recommend that a careful investigation of numts may provide novel insights into the DNA barcoding system with potentially widespread scientific and practical benefits.

\section{Acknowledgement}

We sincerely appreciate Dr. Yanfen Cheng and Wenjia Huang at Nanjing Agricultural University for the sample collection. This work is funded by the National Key R\&D Program of China (2018YFC1200201).

\section{Conflict of interest}

The authors declare that there is no conflict of interests regarding the publication of this paper.

\section{Supplementary Information}

The supplemental material can be downloaded online at: https://stemedicine.org/index.php/stem/article/view/95 


\section{References}

1. Liu J, Ouyang Z, Pimm SL, Raven PH, Wang X, Miao H, et al Protecting China's Biodiversity. Science. 2003, 300:1240-1.

2. He J, Yan C, Marcel H, Wan X, Ren G, Hou Y, et al. Quantifying the effects of climate and anthropogenic change on regiona species loss in China. PLoS One. 2018, 13(7):e0199735.

3. Zheng HR, Cao S. Threats to China's biodiversity by contradictions policy. Ambio. 2015, 44(1):23-33.

4. Daugherty $\mathrm{CH}$, Cree A, Hay JM, Thompson MB. Neglected taxonomy and continuing extinctions of tuatara (Sphenodon). Nature. 1990, 347(6289):177-9.

5. Brisson, J. Aphid wing dimorphisms: linking environmental and genetic control of trait variation. Philos Trans $R$ Soc Lond B Biol Sci. 2010, 365(1540):605-16.

6. Kreuzer M, Howard C, Adhikari B, Pendry CA, Hawkins JA Phylogenomic approaches to DNA barcoding of herba medicines: developing clade-specific diagnostic characters for berberis. Front Plant Sci. 2019, 10:586.

7. JMurugaiyan J, Roesler U. MALDI-TOF MS profiling-advances in species identification of pests, parasites, and vectors. Front Cell Infect Microbiol. 2017, 7:184.

8. Hebert P, Cywinska A. Biological identifications through DNA barcodes. Proc Biol Sci. 2003, 270(1512):313-21.

9. Savolainen V, Cowan RS, Vogler AP, Roderick GK, Lane R. Towards writing the encyclopaedia of life: an introduction to DNA barcoding. Philos Trans R Soc Lond B Biol Sci. 2005 360(1462):1805-11.

10. Haji Bab Aei M, Singer G, Hebert P, Hickey DA. DNA barcoding: how it complements taxonomy, molecular phylogenetics and population genetics. Trends Genet. 2007 23(4):167-72.

11. Matilainen O, Quirós P, Auwerx J. Mitochondria and epigenetics - crosstalk in homeostasis and stress. Trends Cell Biol. 2017, 27(6):453-63.

12. Akhmedov AT, Marín-García J. Mitochondrial DNA maintenance: an appraisal. Mol Cell Biochem. 2015 409(1):283-305

13. Vences $M$, Thomas $M$, Arie $V$, Chiari $Y$, Vieites DR Comparative performance of the 16S rRNA gene in DNA barcoding of amphibians. Front Zool. 2005, 2(1):5.

14. Hebert PD, Ratnasingham S, deWaard JR. Barcoding anima life: cytochrome c oxidase subunit 1 divergences among closely related species. Proc Biol Sci. 2003;270 Suppl 1(Suppl 1):S96-9.

15. Ratnasingham S, Hebert PD. bold: The Barcode of Life Data System (http://www.barcodinglife.org). Mol Ecol Notes. 2007;7(3):355-64

16. Yang C, Xiao Z, Zou Y, Zhang X, Yang B, Hao Y, et al. DNA barcoding revises a misidentification on musk deer Mitochondrial DNA. 2015;26(4):605-12

17. Che J, Chen HM, Yang JX, Jin JQ, Jiang KE, Yuan ZY, et al Universal COI primers for DNA barcoding amphibians. Mo Ecol Resour. 2012, 12(2):247-58.

18. Van D, Ranjit K, Morrow CD, Blanchard EE, Taylor CM, Martin $\mathrm{DH}$, et al. In silico and experimental evaluation of primer sets for species-level resolution of the vaginal microbiota using $16 \mathrm{~S}$ ribosomal RNA gene sequencing. J Infect Dis. 2019, 219(2):305-14.

19.Elbrecht V, Taberlet $P$, Dejean T, Valentini A, Usseglio-Polatera $P$, Beisel JN, et al. Testing the potential of a ribosomal 16S marker for DNA metabarcoding of insects. PeerJ. 2016 4:e1966.

20. Astrin JJ, Huber B, Misof B, Klütsch CFC. Molecular taxonomy in pholcid spiders (Pholcidae, Araneae): evaluation of species identification methods using CO1 and 16S rRNA. Zool Scr. 2006;35(5)

21. Schmitteckert EM, Prokop CM, Hedrich HJJLA. DNA detection in hair of transgenic mice-a simple technique minimizing the distress on the animals. Lab Anim. 1999, 33(4):385.
22. Berner DK, Cavin C, Erper I, Tunali B. First report of anthracnose of mile-a-minute (Persicaria perfoliata) caused by colletotrichum cf. gloeosporioides in Turkey. Plant Dis. 2012, 96(10):1578

23. Srivathsan A, Meier R. On the inappropriate use of Kimura-2parameter (K2P) divergences in the DNA-barcoding literature. Cladistics. 2012, 28:190-194

24. Clare EL, Kerr K, KönigslöW T, Wilson JJ, Hebert PDN. Diagnosing mitochondrial DNA diversity: applications of a sentinel gene approach. J Mol Evol. 2008, 66(4):362-7.

25. Min XJ, Hickey DA. DNA barcodes provide a quick preview of mitochondrial genome composition. PLoS One. 2007;2(3):e325

26. Zhang $A B$, Feng J, Ward RD, Wan $P$, Gao $Q$, Wu J, et al. A new method for species identification via protein-coding and non-coding DNA barcodes by combining machine learning with bioinformatic methods. PLoS One. 2012;7(2):e30986.

27. Austerlitz F, David O, Schaef Fe R B, Bleakley K, Olteanu $\mathrm{M}$, Leblois $\mathrm{R}$, et al. DNA barcode analysis: a comparison of phylogenetic and statistical classification methods. BMC Bioinformatics. 2009, 10 Suppl 14(Suppl 14):S10.

28. Meyer CP, Paulay G. DNA barcoding: error rates based on comprehensive sampling. PLoS Biol. 2005, 3(12):e422.

29. Fišer Pečnikar Ž, Buzan EV. 20 years since the introduction of DNA barcoding: from theory to application. J Appl Genet. 2013;55(1):43-52.

30. Song $H$, Buhay JE, Whiting MF, Crandall KA. Many species in one: DNA barcoding overestimates the number of species when nuclear mitochondrial pseudogenes are coamplified. Proc Natl Acad Sci U S A. 2008, 105(36):13486-91.

31. Thalmann $O$, Hebler J, Poinar HN, Pääbo S, Vigilant L. Unreliable mtDNA data due to nuclear insertions: a cautionary tale from analysis of humans and other great apes. Mol Ecol. 2004, 13(2):321-35

32. Turvey ST, Marr MM, Barnes I, Brace S, Tapley B, Murphy $\mathrm{RW}$, et al. Historical museum collections clarify the evolutionary history of cryptic species radiation in the world's largest amphibians. Ecol Evol. 2019, 9(18):10070-84

33. Kress WJ, Erickson DL. DNA barcodes: methods and protocols. Methods Mol Biol. 2012, 858:3-8.

34. Varadharajan B, Parani M. DMSO and betaine significantly enhance the PCR amplification of ITS2 DNA barcodes from plants. Genome. 2021;64(3):165-71.

35. Moritz C, Dowling TE, Brown WM. Evolution of animal mitochondrial DNA: relevance for population biology and systematics. Annu Rev Ecol Syst. 1987, 18(1):269-92.

36. White DJ, Wolff JN, Pierson M, Gemmell NJ. Revealing the hidden complexities of mtDNA inheritance. Mol Ecol. 2008, 17(23):4925-42

37. Lightowlers RN, Chinnery PF, Turnbull DM, Howell N. Mammalian mitochondrial genetics: heredity, heteroplasmy and disease. Trends Genet. 1997, 13(11):450-5.

38. Jenuth JP, Peterson AC, Shoubridge EA. Tissue-specific selection for different mtDNA genotypes in heteroplasmic mice. Nat Genet. 1997, 16(1):93-5.

39. Li M, SchröDer R, Ni S, Madea B, Stoneking M. Extensive tissue-related and allele-related mtDNA heteroplasmy suggests positive selection for somatic mutations. Proc Natl Acad Sci U S A. 2015, 112(8):2491-6.

40. Berg OG, Kurland CG. Why mitochondrial genes are most often found in nuclei. Mol Biol Evol. 2000;17(6):951-61.

41. Bensasson D, Zhang DX, Hartl DL, Hewitt GM. Mitochondrial pseudogenes: evolution's misplaced witnesses. Trends Ecol Evol. 2001, 16(6):314-21.

42. Ricardo PC, Franoso E, Arias MC. Mitochondrial DNA intraindividual variation in a bumblebee species: A challenge for evolutionary studies and molecular identification. Mitochondrion. 2020, 53:243-54 